\title{
铜催化 2-(2,2-二溴乙烯基)苯酚化合物与苯酚衍生物的串联醚化反应
}

\author{
耿浩兵 $^{a}$ 陈珊珊 ${ }^{a}$ 孙秃 $^{a}$ 张袖丽*, ${ }^{*}$ 王䂞 $*, b$

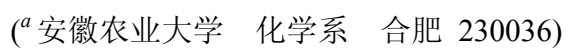 \\ ( ${ }^{b}$ 淮北师范大学 化学系 淮北 235000)
}

\begin{abstract}
摘要 首次报道铜催化 2-(2,2-二溴乙烯基)苯酚类化合物与苯酚衍生物的串联醚化反应. 以 2-(2,2-二溴乙烯基)苯酚衍 生物与苯酚类化合物为起始原料, 醋酸铜为催化剂、碳酸铯作为碱, 在 $N, N$ 二甲基甲酰胺中通过一锅法合成了 24 个未 见文献报道的 2-芳氧基苯并呋喃类化合物. 此合成方法具有原料易得、操作简便、分离纯化简单和无需昂贵配体等优 点.
\end{abstract}

关键词２-(2,2-二溴乙烯基)苯酚; 苯酚; 2-芳氧基苯并呋喃; 二芳醚; 串联反应

\section{Copper-Catalyzed Tandem Etherification Reactions of 2-(2,2-Dibromovinyl)phenol Derivatives with Phenols}

\author{
Geng, Haobing ${ }^{a} \quad$ Chen, Shanshan $^{a} \quad$ Sun, $\mathrm{Xi}^{a} \quad$ Zhang, Xiuli*a $\quad$ Wang, Lei*, \\ ( ${ }^{a}$ Department of Chemistry, Anhui Agricultural University, Hefei 230036) \\ ( ${ }^{b}$ Department of Chemistry, Huaibei Normal University, Huaibei 235000)
}

\begin{abstract}
In this paper, copper-catalyzed tandem etherification reactions of 2-(2,2-dibromovinyl)phenol derivatives with phenols was developed. In the presence of copper acetate as catalyst and cesium carbonate as base, 2-(2,2-dibromovinyl)phenol derivatives reacted with phenols in $N, N$-dimethyl formamide to generate the corresponding 2-phenoxybenzofurans in good yields in one-pot via tandem etherification reactions under ligand-free reaction conditions. Many reaction parameters including bases, copper salts and solvents have been investigated. By screening of bases in the reaction, we found that cesium carbonate is the best one among the tested bases. Experiments shown that strong bases and weak bases, such as potassium hydroxide, potassium $t$-butoxide, potassium acetate and sodium acetate are unfavorable for this reaction. Some common copper catalysts were examined, and the results indicated that copper acetate exhibits the highest reactivity in the reaction. For this reaction, a divalent copper salt is better than a monovalent copper salt. However, $\mathrm{CuO}$ and $\mathrm{Cu}_{2} \mathrm{O}$ gave the poor yield of the product. The solvent also plays a very important role in the reaction. We found that the optimum solvent is $N, N$-dimethyl formamide (DMF). Dimethyl sulfoxide, $N, N$-dimethylacetamide, and $N$-methylpyrrolidone also afforded good product yield. The further investigation revealed that the reaction temperature was one of the factors affecting the yield of the reaction. Scope of the substrate was studied and 24 new 2-aryloxybenzofuran compounds were synthesized in good yields. 2-(4-tert-Butylphenoxy)-5-chlorobenzofuran has the highest reaction yield in 94\%. 4-(Benzofuran-2-yloxy)benzonitrile has the lower yield. Phenol containing an electron-withdrawing group on the benzene ring is difficult to perform the reaction. On the other hand, when an electron-donating group is introduced to 2-(2,2-dibromovinyl)phenol, such as 5-methoxy-, or 4-t-butyl-2-(2,2-dibromovinyl)phenol, the reaction was failed under the present reaction conditions. This method has several advantages including commercial availability of starting materials, simple operation, easy purification of products, and in the absence of ligand. This tandem reaction has the potential applications. It is important to note that the biological activity of the obtained 2-aryloxybenzofuran compounds will be further investigated and will be reported in due course.
\end{abstract}

Keywords 2-(2,2-dibromovinyl)phenol; phenols; 2-phenoxybenzofuran; diaryl ether; tandem reaction

\section{1 引言}

苯并呋喃类化合物是一类在自然界中广泛存在的 天然有机化合物 ${ }^{[1 \sim 5]}$, 它多样的生物活性和巨大的医药 价值，长期以来一直受到人们的关注 ${ }^{[6-9]}$. 作为杂环芳 香化合物研究的热点之一, 已有大量此类化合物被发现
或被合成出来 ${ }^{[10 \sim 13]}$. 长期以来, 有限的天然来源严重限 制了此类化合物的生物活性和药用价值的开发利用，因 此通过合成途径大量获得十分必要. 许多课题组致力于 苯并呋喃类化合物的合成研究 ${ }^{[14]}$. 二芳醚化合物也是 一类在天然产物中大量存在，它具有多样的生物活 性 ${ }^{[15]}$, 也是重要的有机合成中间体. 二芳醚类化合物的

*E-mail: zhxiuli@163.com; leiwang@chnu.edu.cn

Received February 21, 2014; published April 15, 2014.

Supporting information for this article is available free of charge via the Internet at http://sioc-journal.cn.

Project supported by the National Natural Science Foundation of China (Nos. 21372095, 21172092) and Natural Key Project of Education Department, Anhui (No. KJ2013A122).

项目受国家自然科学基金(Nos. 21372095, 21172092)和安徽省教育厅自然科学重点项目(No. KJ2013A122)资助. 
合成方法多样, 其中芳基卤化物与苯酚的乌尔曼偶联反 应为合成二芳醚类化合物的经典方法之一 ${ }^{[16]}$. 乌尔曼 二芳醚合成反应的研究主要是对高反应活性配体的篎 选 ${ }^{[17]}$.

鉴于苯并呋喃类化合物与二芳醚类化合物的生物 活性，通过逆合成分析对 2-芳氧基苯并呋喃类化合物的 结构进行了剖析. 2-芳氧基苯并呋喃可以被分解为 2-溴 苯并呋喃与苯酚, 前者可以由 2-(2,2-二溴乙烯基)苯酚 高效率的生成 ${ }^{[18]}$. 根据 2-(2,2-二溴乙烯基)苯酚的成环 偶联以及与酚的交叉偶联反应 ${ }^{[19,20]}$ 的相关文献的研究, 通过对影响 2-(2,2-二溴乙烯基)苯酚与苯酚衍生物反应 的诸多条件如溶剂、温度、碱和催化剂等的研究, 发现 在醋酸铜作为催化剂, 碳酸铯为碱的条件下, 在 $N, N-$ 二 甲基甲酰胺中能成功催化 2-(2,2-二溴乙烯基)苯酚类化 合物与苯酚衍生物的串联醚化反应，生成 2-苯氧基苯并 呋喃类化合物(图 1).

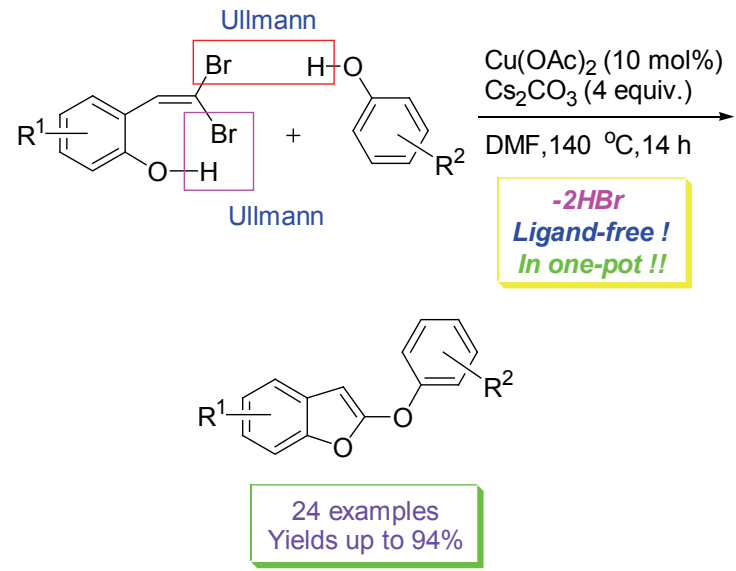

图 1 铜催化 2-(2,2-二溴乙烯基)苯酚衍生物与苯酚的串联醚化反应

Figure 1 Copper-catalyzed tandem etherification reactions of 2-(2,2dibromovinyl)phenol derivatives with phenols

\section{2 结果与讨论}

以 2-(2,2-二溴乙烯基)苯酚(1a)和苯酚 (2a)作为模板 反应, 对反应条件进行优化(表 1). 研究了温度、碱、铜 催化剂和溶剂对反应的影响.

以 $\mathrm{CuI}(10 \% \mathrm{~mol})$ 为催化剂, $\mathrm{K}_{2} \mathrm{CO}_{3}(1.0 \mathrm{mmol})$ 作为 碱, 2-(2,2-二溴乙烯基)苯酚 (1a, $0.25 \mathrm{mmol}$ )和苯酚(2a, $0.30 \mathrm{mmol}$ )在 $N, N$-二甲基甲酰胺(DMF, $2.0 \mathrm{~mL}$ )中于 120 ${ }^{\circ} \mathrm{C}$ 下反应 $14 \mathrm{~h}$, 得到收率为 $41 \%$ 的 2 -苯氧基苯并呋喃 (3a) (表 1, Entry 1), 实验表明, 反应温度是影响反应收 率的因素之一. 当提高反应温度至 $140{ }^{\circ} \mathrm{C}$ 时, 产率提高 到 49\% (表 1, Entry 2).

考察了不同的碱对于反应收率的影响. 结果表明 $\mathrm{Cs}_{2} \mathrm{CO}_{3}$ 是最好的选择, 有 $58 \%$ 的 $3 \mathrm{a}$ 产品收率(表 1 , Entry 3). 当使用更弱的碱 $\mathrm{Na}_{2} \mathrm{CO}_{3}$ 时, 在紫外灯下的硅 胶薄层板上仅能观测到产物的踪迹(表 1, Entry 4). 实验 证明, 强碱对反应不利, 如 $\mathrm{KOH}$ 和 $t-\mathrm{BuOK}$, 收率分别
为 $27 \%$ 和 $46 \%$ (表 1 , Entries 5 和 7). 有趣的是, 钠碱, 如 $\mathrm{NaOH}$ 和 $t$-BuONa，仅能得到目标产物的踪迹(表 1 , Entries 6 和 8). 使用弱碱 $\mathrm{KOAc}$ 和 $\mathrm{NaOAc}$ 时, 没有得到 需要的目标产物(表 1, Entries 9 和 10).

表 1 反应条件的优化

Table 1 Optimization of the reaction conditions ${ }^{a}$
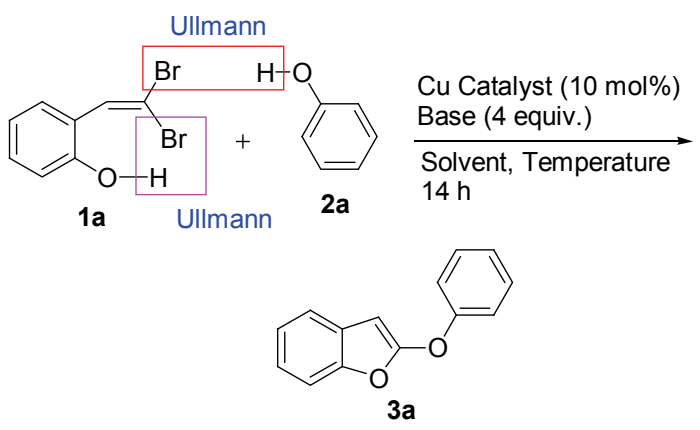

\begin{tabular}{|c|c|c|c|c|}
\hline Entry & Base & $\mathrm{Cu}$ source & Solvent & Yield $^{b} / \%$ \\
\hline $1^{c}$ & $\mathrm{~K}_{2} \mathrm{CO}_{3}$ & $\mathrm{CuI}$ & DMF & 41 \\
\hline 2 & $\mathrm{~K}_{2} \mathrm{CO}_{3}$ & $\mathrm{CuI}$ & DMF & 49 \\
\hline 3 & $\mathrm{Cs}_{2} \mathrm{CO}_{3}$ & $\mathrm{CuI}$ & DMF & 58 \\
\hline 4 & $\mathrm{Na}_{2} \mathrm{CO}_{3}$ & $\mathrm{CuI}$ & DMF & Trace \\
\hline 5 & $\mathrm{KOH}$ & $\mathrm{CuI}$ & DMF & 27 \\
\hline 6 & $\mathrm{NaOH}$ & $\mathrm{CuI}$ & DMF & Trace \\
\hline 7 & $\mathrm{KO} t-\mathrm{Bu}$ & $\mathrm{CuI}$ & DMF & 46 \\
\hline 8 & $\mathrm{NaO} t-\mathrm{Bu}$ & $\mathrm{CuI}$ & DMF & Trace \\
\hline 9 & KOAc & $\mathrm{CuI}$ & DMF & N.R. \\
\hline 10 & $\mathrm{NaOAc}$ & $\mathrm{CuI}$ & DMF & N.R. \\
\hline 11 & $\mathrm{Cs}_{2} \mathrm{CO}_{3}$ & $\mathrm{Cu}(\mathrm{OAc})_{2}$ & $D M F$ & 81 \\
\hline 12 & $\mathrm{Cs}_{2} \mathrm{CO}_{3}$ & $\mathrm{CuCl}_{2}$ & DMF & 68 \\
\hline 13 & $\mathrm{Cs}_{2} \mathrm{CO}_{3}$ & $\mathrm{CuBr}_{2}$ & DMF & 63 \\
\hline 14 & $\mathrm{Cs}_{2} \mathrm{CO}_{3}$ & $\mathrm{CuCl}$ & DMF & 56 \\
\hline 15 & $\mathrm{Cs}_{2} \mathrm{CO}_{3}$ & $\mathrm{CuBr}$ & DMF & 52 \\
\hline 16 & $\mathrm{Cs}_{2} \mathrm{CO}_{3}$ & $\mathrm{CuO}$ & DMF & 49 \\
\hline 17 & $\mathrm{Cs}_{2} \mathrm{CO}_{3}$ & $\mathrm{Cu}_{2} \mathrm{O}$ & DMF & 43 \\
\hline 18 & $\mathrm{Cs}_{2} \mathrm{CO}_{3}$ & $\mathrm{Cu}(\mathrm{OH})_{2}$ & DMF & 65 \\
\hline 19 & $\mathrm{Cs}_{2} \mathrm{CO}_{3}$ & $\mathrm{Cu}\left(\mathrm{NO}_{3}\right)_{2}$ & DMF & 59 \\
\hline 20 & $\mathrm{Cs}_{2} \mathrm{CO}_{3}$ & $\mathrm{Cu}(\mathrm{OAc})_{2}$ & DMSO & 68 \\
\hline 21 & $\mathrm{Cs}_{2} \mathrm{CO}_{3}$ & $\mathrm{Cu}(\mathrm{OAc})_{2}$ & DMAc & 67 \\
\hline 22 & $\mathrm{Cs}_{2} \mathrm{CO}_{3}$ & $\mathrm{Cu}(\mathrm{OAc})_{2}$ & NMP & 64 \\
\hline $23^{d}$ & $\mathrm{Cs}_{2} \mathrm{CO}_{3}$ & $\mathrm{Cu}(\mathrm{OAc})_{2}$ & $\mathrm{CH}_{3} \mathrm{CN}$ & Trace \\
\hline $24^{d}$ & $\mathrm{Cs}_{2} \mathrm{CO}_{3}$ & $\mathrm{Cu}(\mathrm{OAc})_{2}$ & THF & Trace \\
\hline $25^{d}$ & $\mathrm{Cs}_{2} \mathrm{CO}_{3}$ & $\mathrm{Cu}(\mathrm{OAc})_{2}$ & 1,4-Dioxane & Trace \\
\hline $26^{e}$ & $\mathrm{Cs}_{2} \mathrm{CO}_{3}$ & $\mathrm{Cu}(\mathrm{OAc})_{2}$ & DMF & 62 \\
\hline $27^{f}$ & $\mathrm{Cs}_{2} \mathrm{CO}_{3}$ & $\mathrm{Cu}(\mathrm{OAc})_{2}$ & DMF & 37 \\
\hline
\end{tabular}

${ }^{a}$ Reaction conditions: 2-(2,2-dibromovinyl)phenol (1a, $\left.0.25 \mathrm{mmol}\right)$, phenol (2a, $0.30 \mathrm{mmol})$, copper source $(0.025 \mathrm{mmol})$, base $(1.0 \mathrm{mmol})$ and solvent $(2.0 \mathrm{~mL})$ under air at $140{ }^{\circ} \mathrm{C}$ for $14 \mathrm{~h} ;{ }^{b}$ isolated yield; ${ }^{c}$ the reaction temperature was $120{ }^{\circ} \mathrm{C}$; ${ }^{d}$ the reaction temperature was $110{ }^{\circ} \mathrm{C} ;{ }^{e} \mathrm{Cs}_{2} \mathrm{CO}_{3}(0.75$ $\mathrm{mmol}) ;{ }^{f} \mathrm{Cs}_{2} \mathrm{CO}_{3}(0.50 \mathrm{mmol})$. 
对一些常见的催化剂铜盐进行了笁选. 实验证明 $\mathrm{Cu}(\mathrm{OAc})_{2}$ 有最好的反应收率, 为 $81 \%$ (表 1, Entry 11). 对于该体系, 一般二价铜盐的催化效果优于一价铜盐. $\mathrm{CuCl}_{2}$ 和 $\mathrm{CuBr}_{2}$ 都有好的反应收率，产率分别为 $68 \%$ 和 $63 \%$ (表 1, Entries 12 和 13). 实验表明, $\mathrm{CuCl}_{2}$ 有着几乎 和 $\mathrm{Cu}(\mathrm{OAc})_{2}$ 相当的反应收率. 而使用 $\mathrm{CuCl}$ 和 $\mathrm{CuBr}$ 时, 反应产率分别下降到 56\%和 52\% (表 1, Entries 14 和 15). 其它的二价铜盐均有较好的反应收率, 如 $\mathrm{Cu}(\mathrm{OH})_{2}$ 和 $\mathrm{Cu}\left(\mathrm{NO}_{3}\right)_{2}$, 反应产率分别为 65\% 和 59\% (表 1, Entries 18 和 19). $\mathrm{CuO}$ 和 $\mathrm{Cu}_{2} \mathrm{O}$ 的反应效果略差, 仅有 $49 \%$ 和 $43 \%$ 的反应收率(表 1, Entries 16 和 17).

对于乌尔曼二芳醚合成反应，溶剂起着相当重要的 作用. 对于此类反应, 极性非质子性溶剂优于其它类型 的溶剂. 对常用的极性非质子性溶剂做了篮选. 实验证 明， $N, N$-二甲基甲酰胺优于其它溶剂，二甲基亚砜、 $N, N$-二甲基乙酰胺和 $N$-甲基吡咯烷酮也有很好的反应 收率(表 1, Entries 20～22). 乙腈、四氢呋喃和 1,4-二氧 六环的反应效果很差, 在紫外灯下的硅胶薄层板上仅能 观测到产物的踪迹(表 1, Entries 23 和 25). 试图降低 $\mathrm{Cs}_{2} \mathrm{CO}_{3}$ 的用量, 发现低于 4 倍于底物 2-(2,2-二溴乙烯 基)苯酚(1a)的用量, 产率达不到最佳(表 1 , Entries 26 和 27).

通过以上条件实验, 2-(2,2-二溴乙烯基)苯酚衍生物 与苯酚类化合物的反应最优条件确定为: $N, N$-二甲基甲 酰胺作为溶剂, $\mathrm{Cu}(\mathrm{OAc})_{2}(10 \mathrm{~mol} \%)$ 为催化剂, $\mathrm{Cs}_{2} \mathrm{CO}_{3}$ (4.0 equiv.)作为碱, 2-(2,2-二溴乙烯基)苯酚衍生物(1, 1.0 $\mathrm{mmol})$ 和苯酚类化合物 $(2,1.20 \mathrm{mmol})$, 在 $140{ }^{\circ} \mathrm{C}$ 下反应 $14 \mathrm{~h}$.

在确定的最优反应条件下, 我们对反应的底物范围 做了研究, 结果见表 2. 以 2-(2,2-二溴乙烯基)苯酚为反 应底物, 首先对苯酚类化合物(2)的结构对串联反应的 影响作了研究. 考察了 2-(2,2-二溴乙烯基)苯酚与邻位、 间位和对位甲基苯酚的反应. 实验表明，此反应体系对 苯酚类化合物的位阻效应是敏感的. 邻甲基苯酚由于空 间位阻阻碍了反应的进行，使得反应收率降低(表 2 , Entry 4). 这类邻位位阻效应从邻氯苯酚也可以得到进 一步的证实(表 2, Entries 6 和 20). 其次, 也研究了酚类 化合物的电子效应. 在苯酚类化合物的芳环的对位和间 位引入供电子基团时，如 $i$-Pr、 $t$-Bu、 $\mathrm{Me} 、 \mathrm{Et}$ 和 $\mathrm{MeO}$ 等, 该反应的收率明显提高(表 2, Entries 2, 3, 8, 9, 18, 21 和 22). 而在芳环上引入吸电子基团时, 如 $\mathrm{Cl}$ 和强吸电 子的 $\mathrm{CN}$, 串联反应的产率分别下降为 $79 \%$ 和 $42 \%$ (表 2 , Entries 5 和 10). 尤其是, 对硝基苯酚由于强吸电子硝 基的存在, 产物 2-(4-硝基苯氧基)苯并呋喃在硅胶薄层 板上仅能目测到些踪迹, 但是没有能分离出产物. 4-溴 苯酚与 2-(2,2-二溴乙烯基)苯酚的串联反应效果稍差, 反应产率为 $56 \%$ (表 2, Entry 7). $\beta$-萗酚可以得到 $71 \%$ 收 率的目标产物 3k (表 2, Entry 11).
表 2 反应底物的扩展

Table 2 The scope of the substrates

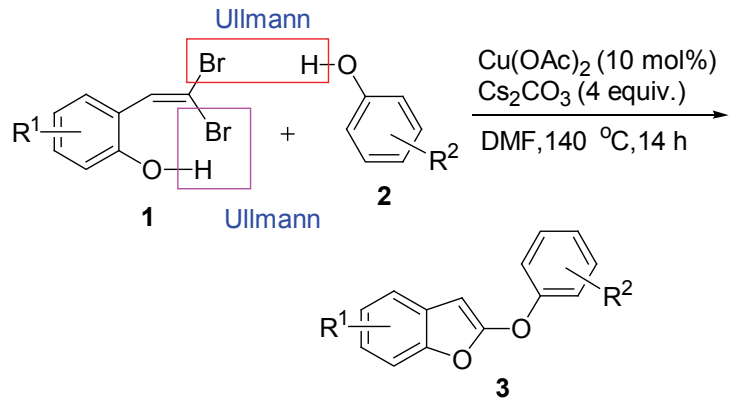

\begin{tabular}{llll}
\hline Entry & Product & 3 & Yield $^{b} \%$ \\
\hline & & & \\
\hline
\end{tabular}

2<smiles>Cc1ccc(Oc2cc3ccccc3o2)cc1</smiles>

3b $\quad 83$

3<smiles>Cc1cccc(Oc2cc3ccccc3o2)c1</smiles>

4<smiles>Cc1ccccc1Oc1cc2ccccc2s1</smiles>

5<smiles>Clc1ccc(Oc2cc3ccccc3o2)cc1</smiles>

3e $\quad 79$

6<smiles>Clc1ccccc1Oc1cc2ccccc2o1</smiles><smiles>Brc1ccc(Oc2cc3ccccc3o2)cc1</smiles>

3 g $\quad 56$

8

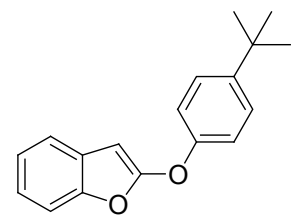




\begin{tabular}{lllll} 
& & & & \\
\hline Entry & Product & 3 & Yield $^{b} / \%$
\end{tabular}

9<smiles>COc1ccc(Oc2cc3ccccc3o2)cc1</smiles>

3i $\quad 87$<smiles>N#Cc1ccc(Oc2cc3ccccc3o2)cc1</smiles>

3j $\quad 42$

11<smiles>c1ccc2cc(Oc3cc4ccccc4o3)ccc2c1</smiles>

12<smiles>Clc1ccc2oc(Oc3ccccc3)cc2c1</smiles>

13<smiles>Brc1ccc2oc(Oc3ccccc3)cc2c1</smiles>

14<smiles>O=[N+]([O-])c1ccc2oc(Oc3ccccc3)cc2c1</smiles><smiles>Brc1cc(Br)c2oc(Oc3ccccc3)cc2c1</smiles>

17<smiles>c1ccc(Oc2cc3c(ccc4ccccc43)o2)cc1</smiles>

18<smiles>CC(C)(C)c1ccc(Oc2cc3cc(Cl)ccc3o2)cc1</smiles>

续表

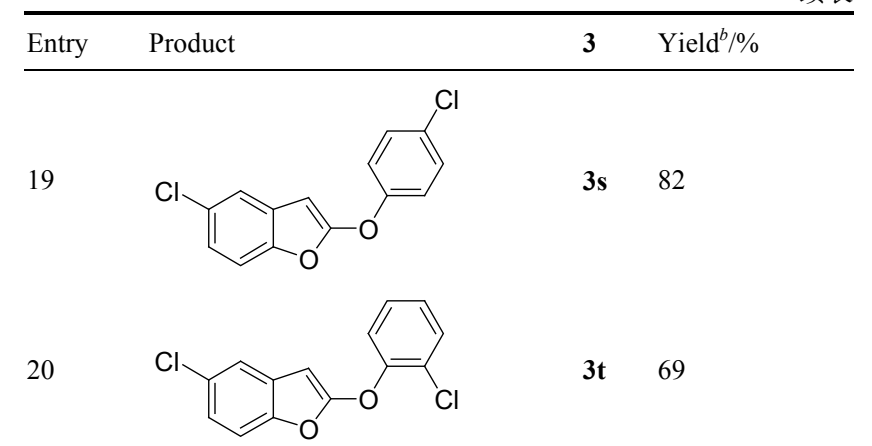

21<smiles>CC(C)c1ccc(Oc2cc3ccccc3o2)cc1</smiles>

3u $\quad 85$

22<smiles>CCc1ccc(Oc2cc3ccccc3o2)cc1</smiles>

23<smiles>Cc1cc(Oc2cc3ccccc3o2)ccc1Cl</smiles>

24<smiles>Cc1cccc(Oc2cc3cc(Cl)ccc3o2)c1</smiles>

25<smiles>COc1ccc2cc(Oc3ccccc3)oc2c1</smiles>

26<smiles>CC(C)(C)c1ccc2oc(Oc3ccccc3)cc2c1</smiles>

3w 73

3x 83

3y 0

$3 z<10$

${ }^{a}$ Reaction conditions: 2-(2,2-dibromovinyl)phenol derivative (1, $\left.0.50 \mathrm{mmol}\right)$, phenol (2, $0.60 \mathrm{mmol}), \mathrm{Cu}(\mathrm{OAc})_{2}(0.050 \mathrm{mmol}), \mathrm{Cs}_{2} \mathrm{CO}_{3}(2.0 \mathrm{mmol})$ and $\mathrm{DMF}$ $(2.0 \mathrm{~mL})$ under air at $140{ }^{\circ} \mathrm{C}$ for $14 \mathrm{~h} ;{ }^{b}$ isolated yield.

尔后我们考察了 2-(2,2-二溴乙烯基)苯酚衍生物 (1)的结构对反应的影响. 当 2-(2,2-二溴乙烯基)苯酚类 化合物的芳环上连有吸电子基团时, 如 $\mathrm{Cl}$ 和 $\mathrm{NO}_{2}$, 该串 联反应的产物收率明显提高 (表 2, Entries 12 和 14). 当 在 2-(2,2-二溴乙烯基)苯酚衍生物的芳环上引入两个卤 素时，反应收率提高更为显著; 同时也没有发现位于 2-(2,2-二溴乙烯基)苯酚衍生物的邻位取代基的位阻效 应(表 2, Entries 15 和 16). 1-(2,2-二溴乙烯基)- $\beta$-萘酚与 苯酚发生串联反应得到 78\%收率的相应产物(表 2 , Entry 
17). 其它底物的串联反应均取得较好的结果(表 2 , Entries 13,19, 23 和 24). 当在 2-(2,2-二溴乙烯基)苯酚类化 合物的芳环引入供电子基团, 如 5-甲氧基和 4-叔丁基 时, 在优化反应条件下该串联反应很难进行(表 2, Entries 25 和 26). 值得提及, 如果在反应体系中没有苯酚 类底物存在下, 仅 2-(2,2-二溴乙烯基)苯酚反应可生成 2-溴苯并呋喃, 产率达 92\%; 所得 2-溴苯并呋喃继续和 苯酚在同样条件下生成 $90 \%$ 收率的 2-苯氧基苯并呋喃.

\section{3 结论}

本文报道了一种以 2-(2,2-二溴乙烯基)苯酚化合物 与苯酚衍生物为起始原料, 通过一锅者串联醚化反应合 成 2-芳氧基苯并呋喃类化合物的方法. 以碳酸铯为碱, 醋酸铜为催化剂, 无任何配体的体系中, 在 $140{ }^{\circ} \mathrm{C}$ 温度 下, 在 $N, N$-二甲基甲酰胺中通过一锅煮实现 2-(2,2-二溴 乙烯基)苯酚类化合物与苯酚衍生物的串联醚化反应, 合成 2-苯氧基苯并呋喃类化合物. 对影响该反应体系的 诸多因素做了研究, 包括碱、铜盐和溶剂等. 对底物适 用范围作了考察, 合成了 24 个未见文献报道的 2-芳氧 基苯并呋喃类化合物. 该反应体系具有操作简便、原料 易得、催化剂价廉及无需添加额外配体等优点, 使得此 串联反应具有潜在的应用价值. 其制备的 2-苯氧基苯并 呋喃类新化合物的生理活性在进一步的研究中.

\section{4 实验部分}

在 $10 \mathrm{~mL}$ 反应管中依次加入 2-(2,2-二溴乙烯基)苯 酚衍生物 $(\mathbf{1}, 0.50 \mathrm{mmol})$ 与苯酚类化合物 $(2,0.60 \mathrm{mmol})$, 醋酸铜 $(0.05 \mathrm{mmol})$, 碳酸铯 $(2.0 \mathrm{mmol}), N, N$-二甲基甲 酰胺 $(2.0 \mathrm{~mL})$, 旋紧旋塞, 置于油浴 $140{ }^{\circ} \mathrm{C}$ 反应 $14 \mathrm{~h}$. 反 应完毕后, 冷却至室温, 加入水, 用乙酸乙酯萃取, 合 并有机相, 用无水硫酸镁干燥, 过滤, 减压浓缩除去溶 剂, 以石油醚与乙酸乙酯按不同比例配制的淋洗剂进行 柱层析分离, 得到目标产物 $\mathbf{3}$, 用 ${ }^{1} \mathrm{H}$ NMR (400 MHz)、

${ }^{13} \mathrm{C} \mathrm{NMR}(100 \mathrm{MHz})$ 和高分辨质谱确定所得产物结构, 表征数据如下.

2-Phenoxybenzofuran (3a): ${ }^{1} \mathrm{H}$ NMR (400 MHz, $\left.\mathrm{CDCl}_{3}\right) \delta: 7.48 \sim 7.40(\mathrm{~m}, 4 \mathrm{H}), 7.26 \sim 7.22(\mathrm{~m}, 5 \mathrm{H}), 5.86$ $(\mathrm{s}, 1 \mathrm{H}) ;{ }^{13} \mathrm{C} \mathrm{NMR}\left(100 \mathrm{MHz}, \mathrm{CDCl}_{3}\right) \delta: 160.08,155.66$, $149.58,129.89,128.91,124.83,123.21,122.80,120.04$, 118.23, 110.74, 83.95. HRMS (ESI) $\left([\mathrm{M}+\mathrm{H}]^{+}\right)$calcd for $\mathrm{C}_{15} \mathrm{H}_{13} \mathrm{O}_{2}: 211.0759$, found 211.0761 .

2-(p-Tolyloxy)benzofuran (3b): ${ }^{1} \mathrm{H}$ NMR (400 MHz, $\left.\mathrm{CDCl}_{3}\right) \delta: 7.44 \sim 7.39(\mathrm{~m}, 2 \mathrm{H}), 7.23 \sim 7.19(\mathrm{~m}, 4 \mathrm{H})$, $7.13 \sim 7.10(\mathrm{~m}, 2 \mathrm{H}), 5.77(\mathrm{~s}, 1 \mathrm{H}), 2.39(\mathrm{~s}, 3 \mathrm{H}) ;{ }^{13} \mathrm{C} \mathrm{NMR}$ $\left(100 \mathrm{MHz}, \mathrm{CDCl}_{3}\right) \delta: 160.73,153.33,149.48,134.58$, $130.32,129.02,123.14,122.57,119.87,118.31,110.66$, 83.03, 20.77. HRMS (ESI) $\left([\mathrm{M}+\mathrm{H}]^{+}\right)$calcd for $\mathrm{C}_{15} \mathrm{H}_{13} \mathrm{O}_{2}$ : 225.0916 , found 225.0914 .
2-(m-Tolyloxy)benzofuran (3c): ${ }^{1} \mathrm{H}$ NMR (400 MHz, $\left.\mathrm{CDCl}_{3}\right) \delta: 7.45 \sim 7.39(\mathrm{~m}, 2 \mathrm{H}), 7.30 \sim 7.22(\mathrm{~m}, 3 \mathrm{H})$, $7.04 \sim 7.02(\mathrm{~m}, 3 \mathrm{H}), 5.84(\mathrm{~s}, 1 \mathrm{H}), 2.38(\mathrm{~s}, 3 \mathrm{H}) ;{ }^{13} \mathrm{C} \mathrm{NMR}$ $\left(100 \mathrm{MHz}, \mathrm{CDCl}_{3}\right) \delta: 160.05,155.49,149.42,140.09$, $129.46,128.83,125.49,123.05,122.59,119.87,118.66$, $115.09,110.62,83.75,21.29$. HRMS (ESI) $\left([\mathrm{M}+\mathrm{H}]^{+}\right)$ calcd for $\mathrm{C}_{15} \mathrm{H}_{13} \mathrm{O}_{2}: 225.0916$, found 225.0913.

2-(o-Tolyloxy)benzofuran (3d): ${ }^{1} \mathrm{H}$ NMR (400 MHz, $\left.\mathrm{CDCl}_{3}\right) \delta: 7.41 \sim 7.39(\mathrm{~m}, 2 \mathrm{H}), 7.31 \sim 7.29(\mathrm{~m}, 1 \mathrm{H})$, $7.25 \sim 7.17(\mathrm{~m}, 4 \mathrm{H}), 7.15 \sim 7.13(\mathrm{~m}, 1 \mathrm{H}), 5.61(\mathrm{~s}, 1 \mathrm{H})$, $2.37(\mathrm{~s}, 3 \mathrm{H}) ;{ }^{13} \mathrm{C}$ NMR $\left(100 \mathrm{MHz}, \mathrm{CDCl}_{3}\right) \delta:$ 160.97, $153.38,149.44,131.64,129.20,129.15,127.25,125.32$, 123.14, 122.38, 119.71, 118.82, 110.59, 81.86, 15.85. HRMS (ESI) $\left([\mathrm{M}+\mathrm{H}]^{+}\right)$calcd for $\mathrm{C}_{15} \mathrm{H}_{13} \mathrm{O}_{2}: 225.0916$, found 225.0913 .

2-(4-Chlorophenoxy)benzofuran (3e): ${ }^{1} \mathrm{H}$ NMR (400 $\left.\mathrm{MHz}, \mathrm{CDCl}_{3}\right) \delta: 7.49 \sim 7.40(\mathrm{~m}, 2 \mathrm{H}), 7.37(\mathrm{~d}, J=8.8 \mathrm{~Hz}$, 2H), $7.27 \sim 7.24(\mathrm{~m}, 2 \mathrm{H}), 7.14(\mathrm{~d}, J=8.8 \mathrm{~Hz}, 2 \mathrm{H}), 5.89$ (s, $1 \mathrm{H}) ;{ }^{13} \mathrm{C} \mathrm{NMR}\left(100 \mathrm{MHz}, \mathrm{CDCl}_{3}\right) \delta: 159.44,154.25$, $149.62,129.98,129.89,128.67,123.34,123.08,120.20$, 119.47, 110.80, 84.52. HRMS (ESI) $\left([\mathrm{M}+\mathrm{H}]^{+}\right)$calcd for $\mathrm{C}_{14} \mathrm{H}_{10} \mathrm{ClO}_{2}: 245.0369$, found 245.0372.

2-(2-Chlorophenoxy)benzofuran (3f): ${ }^{1} \mathrm{H}$ NMR (400 $\left.\mathrm{MHz}, \mathrm{CDCl}_{3}\right) \delta: 7.50(\mathrm{~d}, J=8.0 \mathrm{~Hz}, 1 \mathrm{H}), 7.45 \sim 7.39(\mathrm{~m}$, $2 \mathrm{H}), 7.31 \sim 7.28(\mathrm{~m}, 1 \mathrm{H}), 7.24 \sim 7.18(\mathrm{~m}, 4 \mathrm{H}), 5.79(\mathrm{~s}$, $1 \mathrm{H}) ;{ }^{13} \mathrm{C} \mathrm{NMR}\left(100 \mathrm{MHz}, \mathrm{CDCl}_{3}\right) \delta: 159.40,150.92$, $149.47,130.87,128.66,127.92,125.97,125.05,123.18$, $122.77,119.98,110.67,83.46$. HRMS (ESI) $\left([\mathrm{M}+\mathrm{H}]^{+}\right)$ calcd for $\mathrm{C}_{14} \mathrm{H}_{10} \mathrm{ClO}_{2}: 245.0369$, found 245.0366.

2-(4-Bromophenoxy)benzofuran (3g): ${ }^{1} \mathrm{H}$ NMR (400 $\left.\mathrm{MHz}, \mathrm{CDCl}_{3}\right) \delta: 7.50(\mathrm{~d}, J=8.8 \mathrm{~Hz}, 2 \mathrm{H}), 7.48 \sim 7.39(\mathrm{~m}$, 2H), $7.25 \sim 7.23(\mathrm{~m}, 2 \mathrm{H}), 7.07(\mathrm{~d}, J=8.4 \mathrm{~Hz}, 2 \mathrm{H}), 5.88(\mathrm{~s}$, $1 \mathrm{H}) ;{ }^{13} \mathrm{C} \mathrm{NMR}\left(100 \mathrm{MHz}, \mathrm{CDCl}_{3}\right) \delta: 159.13,154.69$, $149.48,132.75,128.50,123.21,122.99,120.10,119.72$, 117.33, 110.69, 84.56. HRMS (ESI) $\left([\mathrm{M}+\mathrm{H}]^{+}\right)$calcd for $\mathrm{C}_{14} \mathrm{H}_{10} \mathrm{BrO}_{2}$ : 288.9864, found 288.9867 .

2-(4-tert-Butylphenoxy)benzofuran (3h): ${ }^{1} \mathrm{H} \quad \mathrm{NMR}$ $\left(400 \mathrm{MHz}, \mathrm{CDCl}_{3}\right) \delta: 7.49 \sim 7.43(\mathrm{~m}, 4 \mathrm{H}), 7.27 \sim 7.25(\mathrm{~m}$, $2 \mathrm{H}), 7.20 \sim 7.18(\mathrm{~m}, 2 \mathrm{H}), 5.85(\mathrm{~s}, 1 \mathrm{H}), 1.41(\mathrm{~s}, 9 \mathrm{H}) ;{ }^{13} \mathrm{C}$ NMR $\left(100 \mathrm{MHz}, \mathrm{CDCl}_{3}\right) \delta: 160.63,153.29,149.56$, $147.86,129.07,126.74,123.19,122.64,119.96,117.87$, 110.71, 83.33, 34.48, 31.51. HRMS (ESI) $\left([\mathrm{M}+\mathrm{H}]^{+}\right)$calcd for $\mathrm{C}_{18} \mathrm{H}_{19} \mathrm{O}_{2}: 267.1385$, found 267.1386 .

2-(4-Methoxyphenoxy)benzofuran (3i): ${ }^{1} \mathrm{H}$ NMR (400 $\left.\mathrm{MHz}, \mathrm{CDCl}_{3}\right) \delta: 7.43 \sim 7.39(\mathrm{~m}, 2 \mathrm{H}), 7.23 \sim 7.18(\mathrm{~m}, 4 \mathrm{H})$, $6.94(\mathrm{~d}, J=9.0 \mathrm{~Hz}, 2 \mathrm{H}), 5.69(\mathrm{~s}, 1 \mathrm{H}), 3.84(\mathrm{~s}, 3 \mathrm{H}) ;{ }^{13} \mathrm{C}$ NMR $\left(100 \mathrm{MHz}, \mathrm{CDCl}_{3}\right) \delta: 161.56,156.88,149.42$, $148.88,129.10,123.17,122.45,119.98,119.79,114.86$, 110.61, 82.01, 55.68. HRMS (ESI) $\left([\mathrm{M}+\mathrm{H}]^{+}\right)$calcd. for 
$\mathrm{C}_{15} \mathrm{H}_{13} \mathrm{O}_{3}: 241.0865$, found 241.0868.

4-(Benzofuran-2-yloxy)benzonitrile (3j): ${ }^{1} \mathrm{H} \quad \mathrm{NMR}$ $\left(400 \mathrm{MHz}, \mathrm{CDCl}_{3}\right) \delta: 7.68(\mathrm{~d}, J=8.6 \mathrm{~Hz}, 2 \mathrm{H}), 7.53 \sim 7.51$ $(\mathrm{m}, 1 \mathrm{H}), 7.42 \sim 7.39(\mathrm{~m}, 1 \mathrm{H}), 7.29 \sim 7.27(\mathrm{~m}, 2 \mathrm{H}), 7.21(\mathrm{~d}$, $J=8.6 \mathrm{~Hz}, 2 \mathrm{H}), 6.11(\mathrm{~s}, 1 \mathrm{H}) ;{ }^{13} \mathrm{C} \mathrm{NMR}\left(100 \mathrm{MHz}, \mathrm{CDCl}_{3}\right)$ $\delta: 159.19,156.91,149.74,134.14,128.06,123.66,123.43$, $120.53,118.17,117.81,110.88,107.93,87.24$. HRMS (ESI) $\left([\mathrm{M}+\mathrm{H}]^{+}\right)$calcd for $\mathrm{C}_{15} \mathrm{H}_{10} \mathrm{NO}_{2}: 236.0712$, found 236.0708 .

2-(Naphthalen-2-yloxy)benzofuran (3k): ${ }^{1} \mathrm{H} \quad \mathrm{NMR}$ $\left(400 \mathrm{MHz}, \mathrm{CDCl}_{3}\right) \delta: 7.90 \sim 7.86(\mathrm{~m}, 2 \mathrm{H}), 7.79 \sim 7.77(\mathrm{~m}$, $1 \mathrm{H}), 7.57 \sim 7.56(\mathrm{~m}, 1 \mathrm{H}), 7.53 \sim 7.46(\mathrm{~m}, 3 \mathrm{H}), 7.43 \sim 7.38$ $(\mathrm{m}, 2 \mathrm{H}), 7.25 \sim 7.23(\mathrm{~m}, 1 \mathrm{H}), 5.91(\mathrm{~s}, 1 \mathrm{H}) ;{ }^{13} \mathrm{C}$ NMR $(100$ $\left.\mathrm{MHz}, \mathrm{CDCl}_{3}\right) \delta: 159.91,153.22,149.51,133.88,130.77$, $130.00,128.77,127.70,127.31,126.74,125.32,123.11$, $122.74,119.97,118.49,114.03,110.68,84.16$. HRMS (ESI) $\left([\mathrm{M}+\mathrm{H}]^{+}\right)$calcd for $\mathrm{C}_{18} \mathrm{H}_{13} \mathrm{O}_{2}: 261.0916$, found 261.0918 .

5-Chloro-2-phenoxybenzofuran (31): ${ }^{1} \mathrm{H}$ NMR (400 $\left.\mathrm{MHz}, \mathrm{CDCl}_{3}\right) \delta: 7.45 \sim 7.39(\mathrm{~m}, 3 \mathrm{H}), 7.31 \sim 7.16(\mathrm{~m}, 5 \mathrm{H})$, $5.74(\mathrm{~s}, 1 \mathrm{H}) ;{ }^{13} \mathrm{C} \mathrm{NMR}\left(100 \mathrm{MHz}, \mathrm{CDCl}_{3}\right) \delta: 161.37$, $155.18,147.80,130.35,129.99,128.20,125.23,122.80$, 119.64, 118.53, 111.67, 83.09. HRMS (ESI) $\left([\mathrm{M}+\mathrm{H}]^{+}\right)$ calcd for $\mathrm{C}_{14} \mathrm{H}_{10} \mathrm{ClO}_{2}$ : 245.0369, found 245.0366.

5-Bromo-2-phenoxybenzofuran (3m): ${ }^{1} \mathrm{H}$ NMR (400 $\left.\mathrm{MHz}, \mathrm{CDCl}_{3}\right) \delta: 7.55 \sim 7.54(\mathrm{~m}, 1 \mathrm{H}), 7.44 \sim 7.39(\mathrm{~m}, 2 \mathrm{H})$, $7.32 \sim 7.29(\mathrm{~m}, 1 \mathrm{H}), 7.26 \sim 7.23(\mathrm{~m}, 2 \mathrm{H}), 7.21 \sim 7.19(\mathrm{~m}$, 2H), $5.73(\mathrm{~s}, 1 \mathrm{H}) ;{ }^{13} \mathrm{C}$ NMR (100 MHz, $\left.\mathrm{CDCl}_{3}\right) \delta: 161.21$, $155.15,148.17,130.91,129.97,125.52,125.21,122.59$, 118.51, 116.28, 112.14, 82.88. HRMS (ESI) $\left([\mathrm{M}+\mathrm{H}]^{+}\right)$ calcd for $\mathrm{C}_{14} \mathrm{H}_{10} \mathrm{BrO}_{2}$ : 288.9864, found 288.9867.

5-Nitro-2-phenoxybenzofuran (3n): ${ }^{1} \mathrm{H}$ NMR (400 $\left.\mathrm{MHz}, \mathrm{CDCl}_{3}\right) \delta: 8.33 \sim 8.32(\mathrm{~m}, 1 \mathrm{H}), 8.16 \sim 8.13(\mathrm{~m}$, $1 \mathrm{H}), 7.47 \sim 7.43(\mathrm{~m}, 3 \mathrm{H}), 7.31 \sim 7.29(\mathrm{~m}, 1 \mathrm{H}), \quad 7.25 \sim$ $7.23(\mathrm{~m}, 2 \mathrm{H}), 5.84(\mathrm{~s}, 1 \mathrm{H}) ;{ }^{13} \mathrm{C} \mathrm{NMR}\left(100 \mathrm{MHz}, \mathrm{CDCl}_{3}\right) \delta$ : $162.77,154.51,152.25,144.41,130.03,129.44,125.66$, $118.71,118.55,115.89,110.78,83.04$. HRMS (ESI) ([M+ $\mathrm{H}]^{+}$) calcd for $\mathrm{C}_{14} \mathrm{H}_{10} \mathrm{NO}_{4}: 256.0610$, found 256.0608 .

5,7-Dichloro-2-phenoxybenzofuran (3o): ${ }^{1} \mathrm{H}$ NMR $\left(400 \mathrm{MHz}, \mathrm{CDCl}_{3}\right) \delta: 7.46 \sim 7.42(\mathrm{~m}, 2 \mathrm{H}), 7.29 \sim 7.19(\mathrm{~m}$, $5 \mathrm{H}), 5.71(\mathrm{~s}, 1 \mathrm{H}) ;{ }^{13} \mathrm{C}$ NMR $\left(100 \mathrm{MHz}, \mathrm{CDCl}_{3}\right) \delta: 161.95$, $154.71,143.69,131.15,129.98,128.93,125.47,122.70$, $118.59,118.11,116.50,83.08$. HRMS (ESI) $\left([\mathrm{M}+\mathrm{H}]^{+}\right)$ calcd for $\mathrm{C}_{14} \mathrm{H}_{9} \mathrm{Cl}_{2} \mathrm{O}_{2}: 278.9980$, found 278.9985 .

5,7-Dibromo-2-phenoxybenzofuran (3p): ${ }^{1} \mathrm{H} \quad \mathrm{NMR}$ $\left(400 \mathrm{MHz}, \mathrm{CDCl}_{3}\right) \delta: 7.48 \sim 7.41(\mathrm{~m}, 4 \mathrm{H}), 7.29 \sim 7.22(\mathrm{~m}$, $3 \mathrm{H}), 5.72(\mathrm{~s}, 1 \mathrm{H}) ;{ }^{13} \mathrm{C}$ NMR $\left(100 \mathrm{MHz}, \mathrm{CDCl}_{3}\right) \delta: 161.78$, $154.66,145.52$, 131.43, 129.99, 127.78, 125.50, 121.60,
118.63, 116.34, 103.86, 82.88. HRMS (ESI) $\left([\mathrm{M}+\mathrm{H}]^{+}\right)$ calcd for $\mathrm{C}_{14} \mathrm{H}_{9} \mathrm{Br}_{2} \mathrm{O}_{2}: 366.8969$, found 366.8967 .

2-Phenoxynaphtho[1,2-b]furan (3q): ${ }^{1} \mathrm{H}$ NMR (400 $\left.\mathrm{MHz}, \mathrm{CDCl}_{3}\right) \delta: 8.33$ (d, $\left.J=7.8 \mathrm{~Hz}, 1 \mathrm{H}\right), 7.97$ (d, $J=8.0$ $\mathrm{Hz}, 1 \mathrm{H}), 7.79$ (d, $J=9.0 \mathrm{~Hz}, 1 \mathrm{H}), 7.66(\mathrm{~d}, J=9.0 \mathrm{~Hz}, 1 \mathrm{H})$, $7.62(\mathrm{~s}, 1 \mathrm{H}), 7.55 \sim 7.49(\mathrm{~m}, 2 \mathrm{H}), 7.40 \sim 7.36(\mathrm{~m}, 2 \mathrm{H})$, $7.27 \sim 7.22(\mathrm{~m}, 2 \mathrm{H}), 7.17 \sim 7.13(\mathrm{~m}, 1 \mathrm{H}) ;{ }^{13} \mathrm{C}$ NMR $(100$ $\left.\mathrm{MHz}, \mathrm{CDCl}_{3}\right) \delta: 157.88,151.75,142.41,131.95,130.30$, $129.81,128.39,127.10,126.55,126.26,124.68,123.90$, 123.24, 116.81, 116.05, 112.80. HRMS (ESI) $\left([\mathrm{M}+\mathrm{H}]^{+}\right)$ calcd. for $\mathrm{C}_{18} \mathrm{H}_{13} \mathrm{O}_{2}: 261.0916$, found 261.0913.

2-(4-tert-Butylphenoxy)-5-chlorobenzofuran (3r): ${ }^{1} \mathrm{H}$ NMR (400 MHz, $\left.\mathrm{CDCl}_{3}\right) \delta: 7.45(\mathrm{~d}, J=8.6 \mathrm{~Hz}, 2 \mathrm{H})$, $7.40 \sim 7.39(\mathrm{~m}, 1 \mathrm{H}), 7.31 \sim 7.29(\mathrm{~m}, 1 \mathrm{H}), 7.19 \sim 7.16(\mathrm{~m}$, $3 \mathrm{H}), 5.72(\mathrm{~s}, 1 \mathrm{H}), 1.39(\mathrm{~s}, 9 \mathrm{H}) ;{ }^{13} \mathrm{C} \mathrm{NMR}(100 \mathrm{MHz}$, $\left.\mathrm{CDCl}_{3}\right) \delta: 161.88,152.78,148.29,147.76,130.51,128.77$, $126.83,122.62,119.53,118.15,111.61,82.48,34.50$, 31.47. HRMS (ESI) $\left([\mathrm{M}+\mathrm{H}]^{+}\right)$calcd for $\mathrm{C}_{18} \mathrm{H}_{18} \mathrm{ClO}_{2}$ : 301.0995, found 301.0998.

5-Chloro-2-(4-chlorophenoxy)benzofuran $\quad$ (3s): $\quad{ }^{1} \mathrm{H}$ NMR (400 MHz, $\left.\mathrm{CDCl}_{3}\right) \delta: 7.44 \sim 7.39(\mathrm{~m}, 3 \mathrm{H}), 7.31 \sim$ $7.29(\mathrm{~m}, 1 \mathrm{H}), 7.25 \sim 7.15(\mathrm{~m}, 3 \mathrm{H}), 5.74(\mathrm{~s}, 1 \mathrm{H}) ;{ }^{13} \mathrm{C} \mathrm{NMR}$ $\left(100 \mathrm{MHz}, \mathrm{CDCl}_{3}\right) \delta: 161.35,155.16,147.79,130.33$, 129.97, 128.79, 125.21, 122.78, 119.61, 118.52, 111.66, 83.06. HRMS (ESI) $\left([\mathrm{M}+\mathrm{H}]^{+}\right)$calcd for $\mathrm{C}_{14} \mathrm{H}_{9} \mathrm{Cl}_{2} \mathrm{O}_{2}$ : 278.9980 , found 278.9977.

5-Chloro-2-(2-chlorophenoxy)benzofuran $\quad(3 \mathrm{tt}): \quad{ }^{1} \mathrm{H}$ NMR $\left(400 \mathrm{MHz}, \mathrm{CDCl}_{3}\right) \delta: 7.52 \sim 7.50(\mathrm{~m}, 1 \mathrm{H}), 7.38 \sim$ $7.37(\mathrm{~m}, 1 \mathrm{H}), 7.32 \sim 7.29(\mathrm{~m}, 2 \mathrm{H}), 7.25 \sim 7.22(\mathrm{~m}, 2 \mathrm{H})$, $7.18 \sim 7.16(\mathrm{~m}, 1 \mathrm{H}), 5.67(\mathrm{~s}, 1 \mathrm{H}) ;{ }^{13} \mathrm{C}$ NMR $(100 \mathrm{MHz}$, $\left.\mathrm{CDCl}_{3}\right) \delta: 160.75,150.55,147.82,131.08,130.22,128.91$, $128.14,126.50,125.45,122.90,120.55,119.70,111.74$, 82.71. HRMS (ESI) $\left([\mathrm{M}+\mathrm{H}]^{+}\right)$calcd for $\mathrm{C}_{14} \mathrm{H}_{9} \mathrm{Cl}_{2} \mathrm{O}_{2}$ : 278.9980, found 278.9977.

2-(4-iso-Propylphenoxy)benzofuran $(\mathbf{3 u}):{ }^{1} \mathrm{H} \quad \mathrm{NMR}$ $\left(400 \mathrm{MHz}, \mathrm{CDCl}_{3}\right) \delta: 7.46 \sim 7.39(\mathrm{~m}, 2 \mathrm{H}), 7.26(\mathrm{~d}, J=8.4$ $\mathrm{Hz}, 2 \mathrm{H}), 7.24 \sim 7.22(\mathrm{~m}, 2 \mathrm{H}), 7.15(\mathrm{~d}, J=8.4 \mathrm{~Hz}, 2 \mathrm{H})$, $5.80(\mathrm{~s}, 1 \mathrm{H}), 2.99 \sim 2.93(\mathrm{~m}, 1 \mathrm{H}), 1.29(\mathrm{~d}, J=6.8 \mathrm{~Hz}, 6 \mathrm{H})$; ${ }^{13} \mathrm{C}$ NMR $\left(100 \mathrm{MHz}, \mathrm{CDCl}_{3}\right) \delta: 160.67,153.51,149.51$, $145.57,129.04,127.72,123.14,122.59,119.90,118.23$, 110.67, 83.20, 33.57, 24.10. HRMS (ESI) $\left([\mathrm{M}+\mathrm{H}]^{+}\right)$calcd for $\mathrm{C}_{17} \mathrm{H}_{17} \mathrm{O}_{2}: 253.1229$, found 253.1233.

2-(4-Ethylphenoxy)benzofuran (3v): ${ }^{1} \mathrm{H}$ NMR (400 $\left.\mathrm{MHz}, \mathrm{CDCl}_{3}\right) \delta: 7.44 \sim 7.39(\mathrm{~m}, 2 \mathrm{H}), 7.24 \sim 7.21(\mathrm{~m}, 4 \mathrm{H})$, $7.15 \sim 7.13(\mathrm{~m}, 2 \mathrm{H}), 5.78(\mathrm{~s}, 1 \mathrm{H}), 2.68(\mathrm{q}, J=7.6 \mathrm{~Hz}, 2 \mathrm{H})$, 1.27 (t, $J=7.6 \mathrm{~Hz}, 3 \mathrm{H}) ;{ }^{13} \mathrm{C} \mathrm{NMR}\left(100 \mathrm{MHz}, \mathrm{CDCl}_{3}\right) \delta$ : $160.70,153.47,149.49,140.95,129.13,127.63,123.13$, $122.57,119.88,118.30,110.66,83.11,28.21,15.67$. 
HRMS (ESI) $\left([\mathrm{M}+\mathrm{H}]^{+}\right)$calcd for $\mathrm{C}_{16} \mathrm{H}_{15} \mathrm{O}_{2}: 239.1072$, found 239.1068 .

2-(4-Chloro-3-methylphenoxy)benzofuran $\quad(3 \mathbf{w}):{ }^{1} \mathrm{H}$ NMR $\left(400 \mathrm{MHz}, \mathrm{CDCl}_{3}\right) \delta: 7.49 \sim 7.47(\mathrm{~m}, 1 \mathrm{H}), 7.43 \sim$ $7.41(\mathrm{~m}, 1 \mathrm{H}), 7.36(\mathrm{~d}, J=8.6 \mathrm{~Hz}, 1 \mathrm{H}), 7.27 \sim 7.25(\mathrm{~m}$, $2 \mathrm{H}), 7.10 \sim 7.09(\mathrm{~m}, 1 \mathrm{H}), 7.01 \sim 6.98(\mathrm{~m}, 1 \mathrm{H}), 5.88(\mathrm{~s}$, $1 \mathrm{H}), 2.41(\mathrm{~s}, 3 \mathrm{H}) ;{ }^{13} \mathrm{C}$ NMR $\left(100 \mathrm{MHz}, \mathrm{CDCl}_{3}\right) \delta: 159.69$, $154.05,149.61,137.94,130.16,130.13,128.77,123.30$, 122.97, 120.50, 120.15, 116.87, 110.80, 84.32, 20.29. HRMS (ESI) $\left([\mathrm{M}+\mathrm{H}]^{+}\right)$calcd for $\mathrm{C}_{15} \mathrm{H}_{12} \mathrm{ClO}_{2}: 259.0526$, found 259.0527 .

5-Chloro-2-(m-tolyloxy)benzofuran (3x): ${ }^{1} \mathrm{H} \quad \mathrm{NMR}$ $\left(400 \mathrm{MHz}, \mathrm{CDCl}_{3}\right) \delta: 7.39(\mathrm{~d}, J=2.0 \mathrm{~Hz}, 1 \mathrm{H}), 7.32 \sim 7.28$ $(\mathrm{m}, 2 \mathrm{H}), 7.18 \sim 7.16(\mathrm{~m}, 1 \mathrm{H}), 7.07 \sim 7.01(\mathrm{~m}, 3 \mathrm{H}), 5.73(\mathrm{~s}$, $1 \mathrm{H}), 2.40(\mathrm{~s}, 3 \mathrm{H}) ;{ }^{13} \mathrm{C} \mathrm{NMR}\left(100 \mathrm{MHz}, \mathrm{CDCl}_{3}\right) \delta: 161.50$, $155.15,147.78,140.33,130.41,129.66,128.76,126.00$, $122.71,119.58,119.11,115.49,111.65,82.98,21.38$. HRMS (ESI) $\left([\mathrm{M}+\mathrm{H}]^{+}\right)$calcd for $\mathrm{C}_{15} \mathrm{H}_{12} \mathrm{ClO}_{2}: 259.0526$, found 259.0523 .

\section{References}

[1] Hu, X.; Wu, J.-W.; Wang, M.; Yu, M.-H.; Zhao, Q.-S.; Wang, H.-Y.; Hou, A.-J. J. Nat. Prod. 2012, 75, 82.

[2] Liu, Y. H.; Kubo, M. K.; Fukuyama, Y. J. Nat. Prod. 2012, 75, 2152.

[3] Wu, S.-F.; Chang, F.-R.; Wang, S.-Y.; Hwang, T.-L.; Lee, C.-L.; Chen, S.-L.; Wu, C.-C.; Wu, Y.-C. J. Nat. Prod. 2011, 74, 989.

[4] Coy-Barrera, E. D.; Cuca-Suárez, L. E.; Sefkow, M. Phytochemistry 2009, 70, 1309.

[5] Li, Y.-P.; Dong, L.-B.; Chen, D.-Z.; Li, H.-M.; Zhong, J.-D.; Li, F.; Liu, X.; Wang, B.; Li, R.-T. Phytochem. Lett. 2013, 6, 281.

[6] He, Y.-T.; Xu, J.; Yu, Z.-H.; Gunawan, A.-M.; Wu, L.; Wang, L. Zhang, Z.-Y. J. Med. Chem. 2013, 56, 832.

[7] (a) Telvekar, V. N.; Belubbi, A.; Bairwa, V. K.; Satardekar, K. Bioorg. Med. Chem. Lett. 2012, 22, 2343. (b) Ryu, C.-K; Song, A.-L.; Lee, J.-Y.; Hong, J.-A.; Yoon, J.-H.; Kim, A. Bioorg. Med. Chem. Lett. 2010, 20, 6777. (c) Sidhu, P. S.; AbdelAziz, M. H.; Sarkar, A.; Mehta, A. Y.; Zhou, Q. B.; Desai, U. R. J. Med. Chem. 2013, 56, 5059 .

[8] He, Y. T.; Xu, J.; Yu, Z.-H.; Gunawan, A. M.; Wu, L.; Wang, L.; Zhang, Z.-Y. J. Med. Chem. 2013, 56, 832.
[9] Gunosewoyo, H.; Midzak, A.; Gaisina, I. N.; Sabath, E. V.; Fedolak, A.; Hanania, T.; Brunner, D.; Papadopoulos, V.; Kozikowski, A. P. J. Med. Chem. 2013, 56, 5115.

[10] Millot, M.; Kaouadji, M.; Champavier, Y.; Gamond, A.; Simon, A.; Chulia, A. J. Phytochem. Lett. 2013, 6, 31 .

[11] (a) Schneider, B. Phytochemistry 2003, 64, 459. (b) Akgul, Y. Y.; Anil, H. Phytochemistry 2003, 63, 939.

[12] Liu, Z.-X.; Xia, Y.; Zhou, S.-Y.; Wang, L.; Zhang, Y.; Wang, J.-B. Org. Lett. 2013, 15, 5032.

[13] (a) Yuan, H.; Bi, K.-J.; Li, B.; Yue, R.-C.; Ye, J.; Shen, Y.-H.; Jin, H.-Z.; Sun, Q.-Y.; Zhang, W.-D. Org. Lett. 2013, 15, 4742. (b) Qi, J.-P.; Cao, D.-R. Acta Chim. Sinica 2010, 68, 2019. (元金萍，曹德 榕, 化学学报, 2010, 68, 2019.) (c) Zhao, Y.-H.; Liu, W.-J.; Sun, X.-W.; Lin, G.-Q. Chin. J. Org. Chem. 2012, 32, 1919. (赵云辉, 刘 文杰，孙兴文，林国强，有机化学，2012，32，1919.) (d) Gao, W.-T.; Jiang, Y.; Li, Y.; Li, F.; Yan, Y. Chin. J. Chem. 2012, 30, 822. (e) Fang, H.-B.; Jin, L.; Huang, N.-Y.; Wang, J.-Z.; Zou, K.; Luo, Z.-G. Chin. J. Chem. 2013, 31, 831. (f) Zhu, C.-L.; Yang, L.-J.; Nie, J.; Zheng, Y.; Ma, J.-A. Chin. J. Chem. 2012, 30, 2693. (g) Jin, Q.; Xin, M.-H.; Cong, X.; You, D.-Q. Chin. J. Org. Chem. 2013, 33, 590. (金秋，辛敏行，丛欣，尤启冬，有机化学, 2013, 33, 590.)

[14] (a) Anxionnat, B.; Pardo, D. G.; Ricci, G.; Rossen, K.; Cossy, J. Org. Lett. 2013, 15, 3876. (b) Yamaguchi, M.; Katsumata, H.; Manabe, K. J. Org. Chem. 2013, 78, 9270. (c) Kuram, M. R.; Bhanuchandra, M.; Sahoo, A. K. Angew. Chem., Int. Ed. 2013, 52, 4607 (d) Zeng, W.; Wu, W.-Q.; Jiang, H.-F.; Huang, L.-B.; Sun, Y.-D. Chen, Z.-W.; Li, X.-W. Chem. Commun. 2013, 49, 6611. (e) Zhu, R.-Y.; Wei, J.-B.; Shi, Z.-J. Chem. Sci. 2013, 4, 3706. (f) Liu, J.-M.; Zhang, N.-F.; Yue, Y.-Y.; Wang, D.; Zhang, Y. L.; Zhang, X.; Zhuo, K.-L. RSC Advance 2013, 3, 3865. (g) Zhou, L.; Shi, Y.; Xiao, Q.; Liu, Y.-Z.; Ye, F.; Zhang, Y.; Wang, J.-B. Org. Lett. 2011, 13, 968.

[15] (a) Delfín, D. A.; Morgan, R. E.; Zhu, X. H.; Werbovetz, K. A. Bioorg. Med. Chem. 2009, 17, 820. (b) Bryant, V. C.; Kumar, K.; Nyong, A. M.; Natarajan, A. Bioorg. Med. Chem. Lett. 2012, 22, 245. (c) Asakawa, Y. A.; Ludwiczuk, A.; Nagashima, F. Phytochemistry 2013, 91, 52. (d) Lv, J.-J.; Xu, M.; Wang, D.; Zhu, H. T.; Yang, C.-R.; Wang, Y. F.; Li, Y.; Zhang, Y.-J. J. Nat. Prod. 2013, $76,926$.

[16] (a) Ullmann, F.; Sponagel, P. Chem. Ber. 1905, 38, 2211. (b) Monnier, F.; Taillefer, M. Angew. Chem., Int. Ed. 2009, 48, 6954.

[17] (a) Ma, D.-W.; Cai, Q. Org. Lett. 2003, 5, 3799. (b) Salih, M. Q.; Beaudry, C. M. Org. Lett. 2013, 15, 4540. (c) Uchiro, H.; Kato, R.; Arai, Y.; Hasegawa, M.; Kobayakawa, Y. Org. Lett. 2011, 13, 6268. (d) Niu, J.-J.; Zhou, H.; Li, Z.-G.; Xu, J.-W.; Hu, S. J. J. Org. Chem. 2008, 73, 7814. (e) Collins, J. C.; Farley, K. A.; Limberakis, C.; Liras, S.; Price, D.; James, K. J. Org. Chem. 2012, 77, 11079.

[18] (a) Chen, W.; Zhang, Y.-C.; Zhang, L.; Wang, M.; Wang, L. Chem. Commun. 2011, 47, 10476. (b) Ji, Y.; Li, P.-H.; Zhang, X.-L.; Wang, L. Org. Biomol. Chem. 2013, 11, 4095.

[19] Xia, Z.-M.; Wang, K.; Zheng, J.-N.; Ma, Z.-Y.; Jiang, Z.-G.; Wang, X.-X.; Lv, X. Org. Biomol. Chem. 2012, 10, 1602.

[20] Jouvin, K.; Bayle, A.; Legrand, F.; Evano, G. Org. Lett. 2012, 14, 1652 .

[21] Newman, S. G.; Aureggi, A.; Bryan, C. S.; Lautens, M. Chem. Commun. 2009, 5236. 